\title{
Living Reviews - Innovative Resources for Scholarly Communication Bridging Diverse Spheres of Disciplines and Organisational Structures
}

\author{
Claus Dalchow ${ }^{1}$, Michael Nentwich ${ }^{2}$, Patrick Scherhaufer ${ }^{3}$, Christina Weyher ${ }^{4}$ \\ ${ }^{1}$ Leibniz-Centre for Agricultural Landscape Research \\ Eberswalder Strasse 84, 15374 Müncheberg, Germany \\ e-mail: cdalchow@zalf.de \\ ${ }^{2}$ Institute of Technology Assessment (ITA), Austrian Academy of Sciences \\ Strohgasse 45, 1030 Vienna, Austria \\ e-mail:mnent@oeaw.ac.at \\ ${ }^{3}$ Department of Political Science, Institute for Advanced Studies (IHS) \\ Stumpergasse 56, 1060 Vienna, Austria \\ e-mail: patrick.scherhaufer@ihs.ac.at \\ ${ }^{4}$ Heinz Nixdorf Center for Information Management, Max Planck Society \\ Boltzmannstrasse 2, 85748 Garching, Germany \\ e-mail: christina.weyher@zim.mpg.de
}

\begin{abstract}
This contribution presents the concept and analyses the path of diffusion of an innovative publishing idea that originated in one speciality in physics and is now about to spread into other fields, including the social sciences. We discuss the conditions of success and fostering as well as hampering factors on the road for what we consider to be a truly revolutionary concept of presenting the state of knowledge in potentially any given academic discipline. First, we will present the overall idea of Living Reviews (www.livingreviews.org), which are open access online journals featuring an innovative editorial concept for the publication of high-quality scientific content. Second, we discuss the proliferation of this unique concept. Finally, we shall aim at summarising lessons learned from our shared experience in establishing these publishing ventures in our respective fields. Our conclusions give hints for potential future "cyber-activists" who are considering setting up further Living Review projects.
\end{abstract}

Keywords: state-of-the-art articles; living reviews; review articles; physics; social sciences

\section{Introduction}

Living Reviews journals publish commissioned, peer-refereed review articles that are regularly being updated by their authors - which is the significance of the word 'living' in the journal titles. The first journal, Living Reviews in Relativity, went online in 1998. To date, the Living Reviews family consists of three running journals and another one in an advanced stage of preparation [1]. More journals are in the course of formation. All journals are offered as a free service to the scientific community.

\subsection{Editorial Concept}

Living Reviews journals publish - as the name suggests - review articles. The reviews provide current and insightful overviews of what is happening in a field of research. They highlight significant issues affecting the field; provide evaluative commentary on the essential techniques and concepts being used in current research; and offer insight into the challenges facing future research efforts. Through their commentary articles will also guide users to the key literature and online resources pertaining to these fields. The reviews are solicited by an international editorial boards from experts in the fields. The reviews can either be umbrella articles that have a relatively wide scope, or more specific articles that develop one line of research in some depth [2]. The envisioned target group is drawn from the entire scientific community: graduate students may use the journals as a starting point for their initial literature surveys and as a way to learn about fields peripheral to their own; researchers use the journal to find the latest results in fields outside of their current awareness, to track down bibliographic references that they have not recorded, or even to uncover ways in which their skills can be applied in a new field; and lecturers use the journal to find information and visual materials appropriate for presentations.

The key feature of Living Reviews is the fact that its articles are maintained by their authors. Periodically updates of a review are being published covering the latest important research developments. 
An update is a substantial revision of the article. When updating the article authors will build on the text of the original article. They are free to rewrite individual sections, add new material to their articles, or rework the entire piece. The requirement from our side is that the changes will reflect the progress in the field. From the editorial point of view, an update is treated like a new publication and is given full referee scrutiny. It is published with a new publication number and accessible under a new URL. The preceding version remains accessible at its original location on the web server. To keep the article's evolution transparent to the reader, a history file (see Fig. 1) records and summarises the revisions an article underwent.

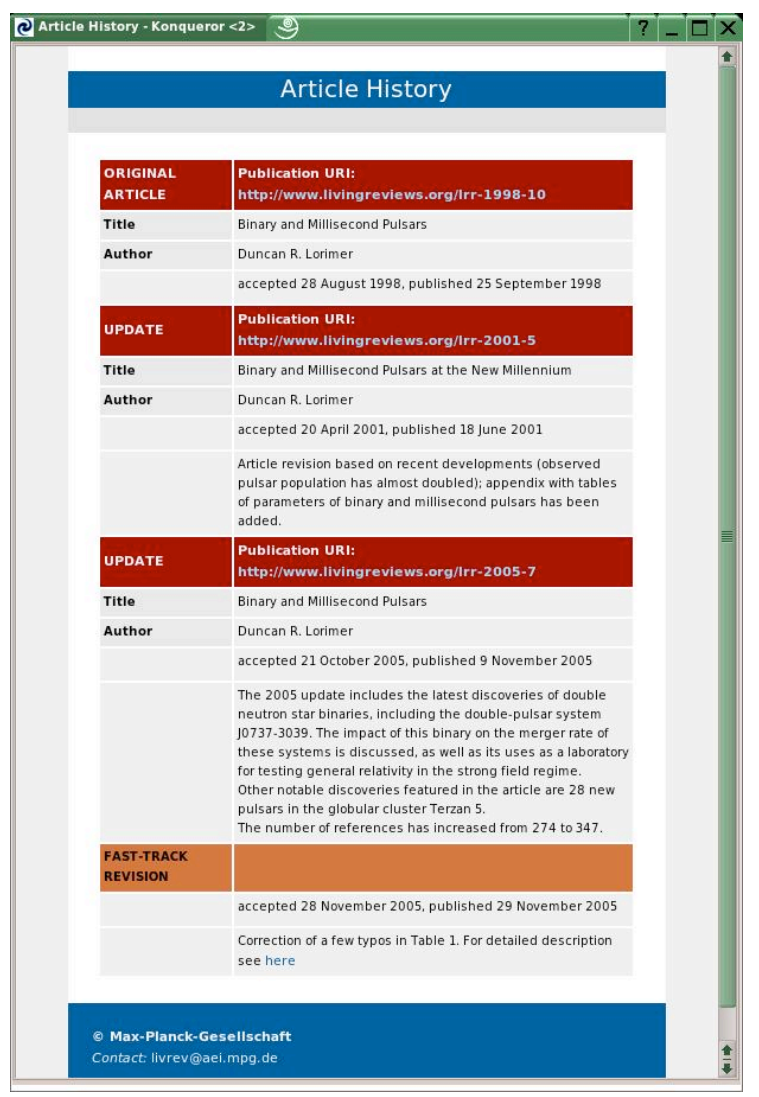

Figure 1: Example of an article history file

The more intricate questions that are implicit to this 'living' concept became only obvious after a few years of gathering experience. For instance, an author may want to take on a co-author for writing the update of his article (cf. www.livingreviews.org/lrr-2003-2) or an author retires completely and wants to hand over the responsibility for the article's maintenance to a new author. There are also cases when a given subject becomes too broad to be covered in one review, therefore the article could be split in future revisions. Or the subject progresses in a way that makes a complete shift of the article's focus necessary. No general rules can be applied here yet, and we are still learning about the ways in which an article can evolve. The topic editor, the editorial team and the authors deal with such issues on a case-by-case basis.

As the reviews are updated periodically, they are more up-to-date than subsequent editions of classic textbooks. However, experience shows that the initial goal to have articles updated about every two years seems too ambitious. Many authors use this feature very actively, but a considerable number of reviews published in the first years (in LRR) are still awaiting a major revision.

Particularly for authors working in very active research areas there is a danger of a backlog of new developments building up over a couple of years. The experience has shown that with each year the threshold to start writing an update becomes higher. It took several years and many intense discussions with editorial board members and authors alike to find a satisfying solution, which would make the intervals between updates more predictable and the work for authors more manageable. To that end a more lightweight form of amendments was devised, which would allow authors to update their review by easy stages in between major overhauls. Two requirements needed to be considered: First, since these small-scale revisions would not be treated like a full new publication, any changes in the current article needed to be recorded and documented in detail. Second, the 
organizational and technical effort needed to be kept manageable for authors and editorial team. Finally, the socalled Fast-Track-Revisions (FTR) were introduced in 2005.

They should be used by authors to make small-scale amendments, like annual updates to their article, but can also be utilized for classical errata. The changes made in a FTR are reviewed by the responsible topic editor to ensure that the high quality of the reviews is hold up. Unlike major updates, a FTR replaces the preceding version of an article. All changes in the article are implemented using a special macro and are described in detail by the author. Thus it is possible for the reader to see exactly where changes occurred. (Technically, the LaTeX source format of our articles allows the running of text-based diffs, which guarantees that only changes that are properly marked will get through.) An example for the implementation of a FTR can be found in www.livingreviews.org/lrr-2004-4 - a fast track revision to add recent developments, published 15 months after the original publication (see also Fig. 2). In www.livingreviews.org/lrr-2005-7 the FTR is used to publish an erratum.

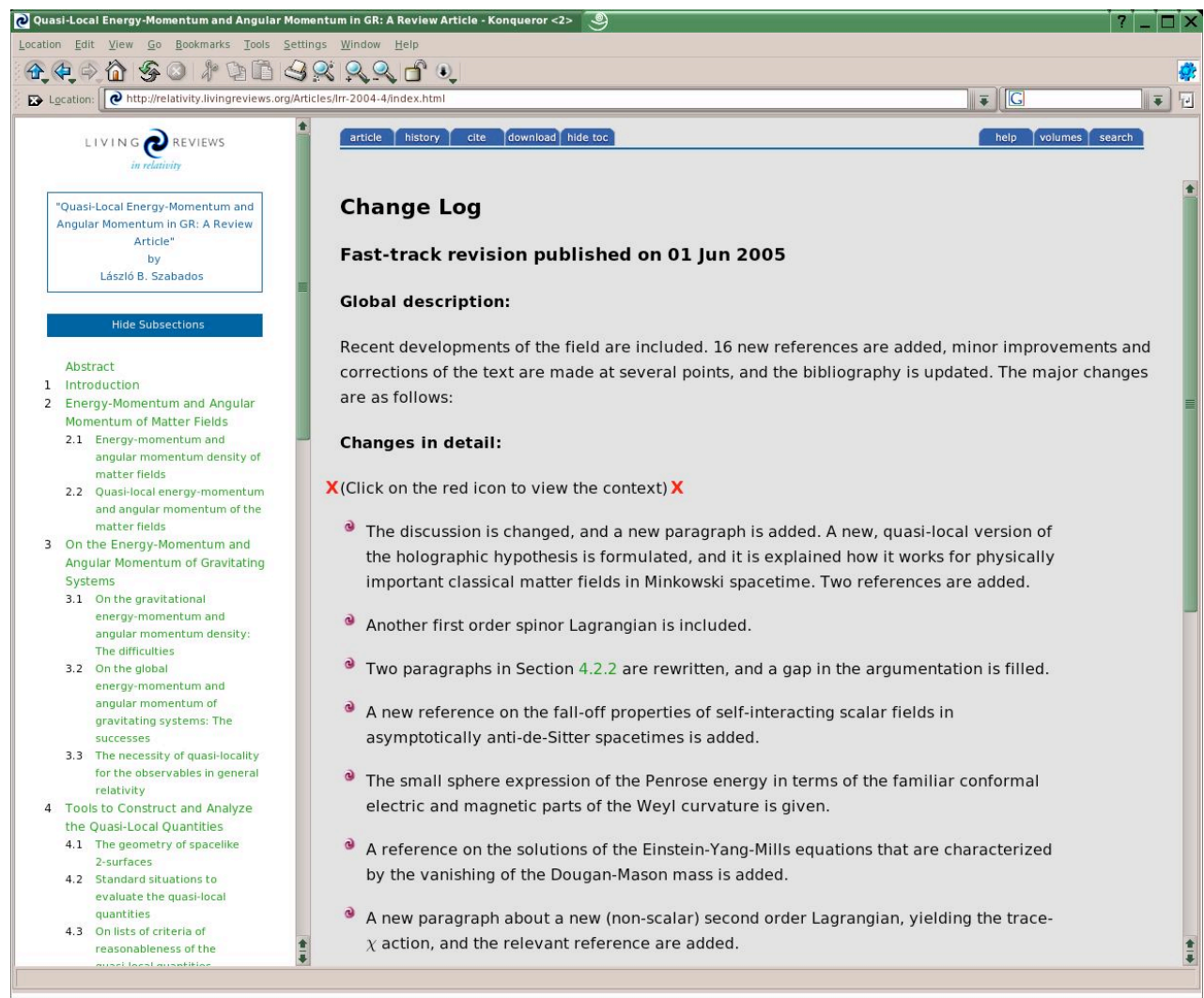

Figure 2: Change Log for Fast-Track-Revision

\subsection{Online Features}

The journals provide various sophisticated web presentation and online functionalities, as developed and maintained by the project. The style of use for electronic documents puts certain requirements on the journal's presentation and content. Different from linear reading of a printed text, users navigating through a hypertext document may follow a very individual path while assimilating information. They may suspend their own reading of an article to follow up a link to an online resource, to another section of the article, to a reference, or to an index. These jumps may lead to further links. We seek to support such non-linear access in various ways: the article interface is designed for easy navigation through the whole article. References can be tracked throughout the text, which allows users to follow the discussion of a given reference easily. Popup windows are used to let users view referenced images or equations without the need to leave the reading context (see Fig. 3). Authors are also asked to keep the online accessibility in mind when writing their review, e.g. by organizing the article in as far as possible self-contained sections and subsections that are not too long and thus suitable to be read on a computer screen. 


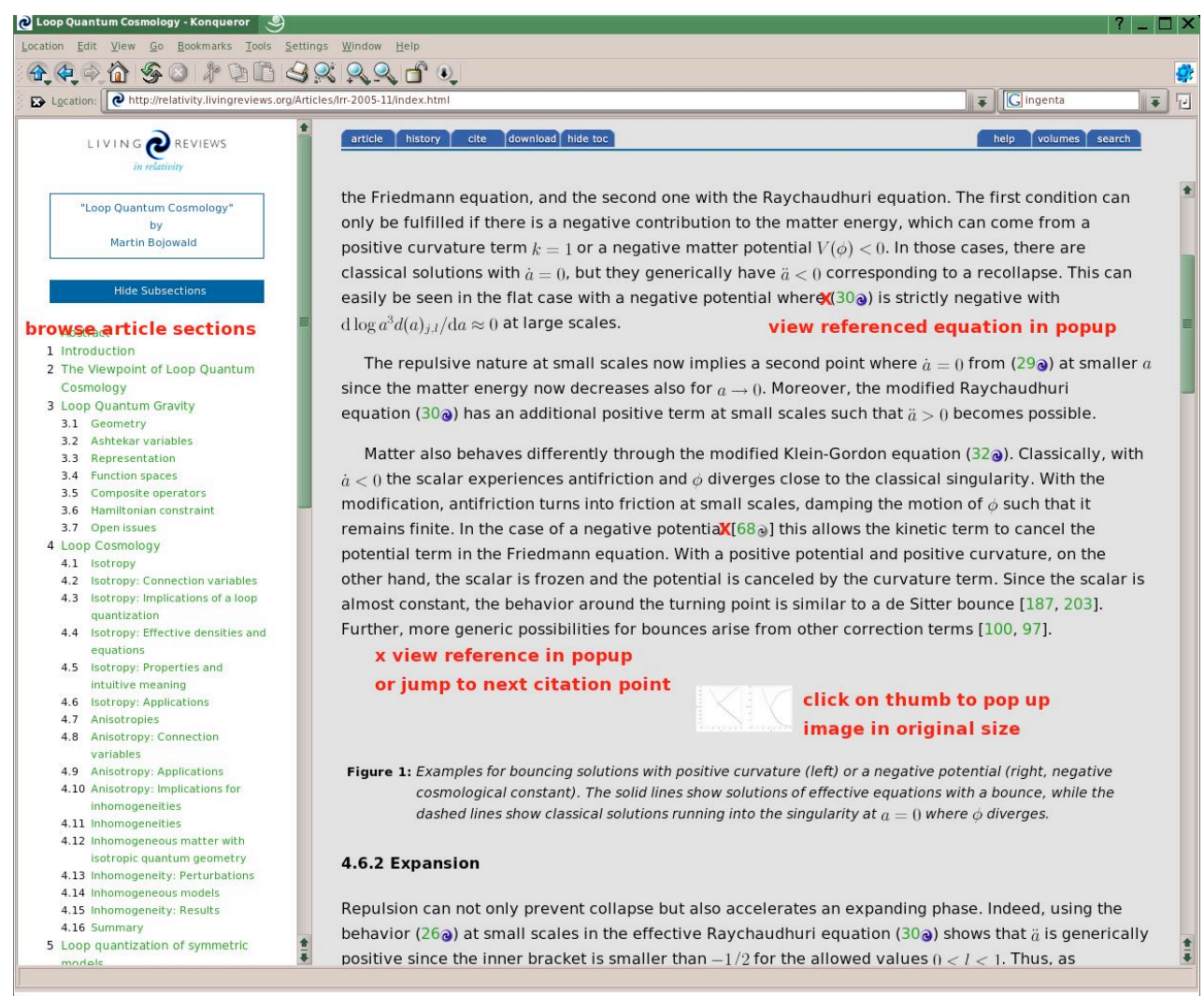

Figure 3: Article Interface

The online format makes any restrictions concerning the length of an article irrelevant. Articles may also include an unlimited number of references, images, figures, tables, animations or movies - and we encourage our authors to make generous use of these possibilities. Even program code can be attached to an article, to allow a user the reproduction of described results. The HTML format of an article is considered the authoritative version, containing the richest features and metadata. A user may however download PDF versions of the article for printout, as well as the complete bibliography in a database file, or any attached movies in a compressed archive.

The extensive bibliographies of the articles (some containing up to 400 references) provide a very rich information resource, as they point readers to the most important literature in the field. To extend the usability of this resource, all references cited in Living Reviews articles are collated in an online searchable reference database, building up over time a comprehensive, annotated bibliography of current research.

Database search results can be exported in various formats, like BibTeX, XHMTL, XML or RDF-DC. Since all records in the database are cross-linked back to the citing article, a user may directly jump from a search result to its citing context (see Fig. 4). Through this, a user obtains not only the information that a given reference was cited, but can immediately check how it was evaluated by the author of the review. Currently, the reference databases of LRR and LRSP contain a total of about 13,000 bibliographic records.

All software for the publication and management of the journals - the ePublishing Toolkit - has been developed by the Living Reviews team as an open source project [3]. Some of these tools are also useful for applications outside of a journal context (e.g. a scientist could use the PublicationBuilder component to put a paper with LR functionalities online on his homepage), and we welcome reuse or collaborative development. 


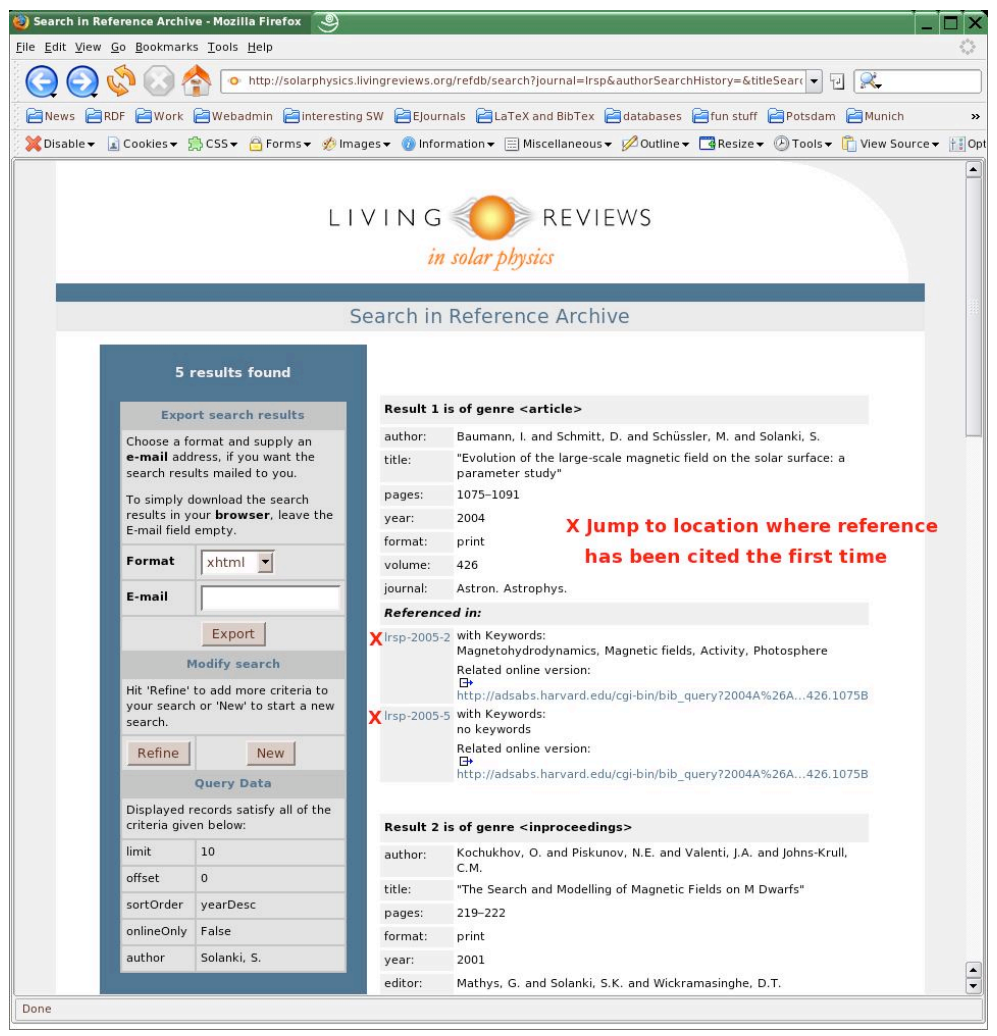

Figure 4: Reference Database Search Results

\section{Spreading the Concept}

In 2001, Living Reviews was selected as a reference project by the then newly funded Heinz Nixdorf Center for Information Management in the Max Planck Society (ZIM, [4]), with the goal to facilitate the foundation of new Living Reviews journals in further areas of research. The Living Reviews project provides a central publishing platform [5] for all institutions interested in setting up such a journal. Additionally, it provides editorial services to Max Planck Institutes that are publishing a Living Reviews journal.

The core requirements for starting up a journal are: (i) it has to be in accordance with the editorial concept of peer-refereed, updated review articles, commissioned by an international editorial board; (ii) the development of a sound scientific concept for the new journal; (iii) its publication as open access service; and (iv) the maintenance of a high quality web presentation. In the future, a Living Reviews consortium representing all members of the journal family plus funding bodies, will oversee the further development of the project and the foundation of new journals. The appropriate organisational embedding and the scope of responsibility for this consortium are currently being defined.

In the following sections we will describe the different already existing journals. Although rather similar in technical performance and in concentration on review articles, the variety in scope and of institutional affiliation is broad.

\subsection{Living Reviews in Physics}

Living Reviews in Relativity (LRR, relativity.livingreviews.org, published by the Max Planck Institute for Gravitational Physics) as the 'prototype' journal was started back in 1996 by scientists of the Max Planck Institute for Gravitational Physics (Albert Einstein Institute, AEI). Several factors helped to shape the initial journal concept [6]: physics has been a pioneering field in using electronic means for scholarly communication, and much of the current research was already available in document repositories (mainly the arXiv e-print archive [7]). Reviews to evaluate research findings were an established part of the scientific communication culture - though they were far too slow for a rapidly evolving, interdisciplinary field like gravitational physics and did not cover electronic resources. (In traditional review journals in physics, a given subject field has only a chance of being reviewed every ten to twenty years.) Finally, new web-based technologies significantly lowered 
the barriers to starting a publishing project, and first small-scale publishers arose from academic environments. After two years of preparation, the formation of the initial editorial board, the commissioning of a suite of about 20 articles, and the development of the first generation of publishing tools, volume one went online in January $1998[8]$.

Within the first years, a major challenge was to transform the enthusiasm of the start-up phase into a running editorial management, and to ensure a steady article flow. To improve the coverage of individual subject fields, the concept of topic editors was introduced. Topic editors are members of the editorial board who are responsible for the adequate and comprehensive coverage in their specific area of expertise. They help to avoid delays in the invitation phase, which can arise when potential authors are not sure what exactly they are expected to deliver, how free they are to modify the topic or to cut it down, or if it seems too huge to be reviewed with reasonable investment of time and energy. The topic editor will negotiate such questions directly with a potential author. They are also responsible for suggesting referees and will, if necessary, step in to resolve conflicts in a refereeing issue. Last but not least, they will guide the evolution of an article throughout its lifetime.

Much effort in the first years of LRR went also into promotion activities. The journal was regularly presented at major relativity conferences, which helped a lot to gain attention in the community. Since then LRR has become a great success and a standard reference in the field. Citation rates and journal web statistics confirm an impressive outreach in the target community (see Table 1). The journal has been acknowledged with 'Recognized Journal Status' by the European Physical Society, and only recently it has been accepted for inclusion in the Science Citation Index.

\begin{tabular}{|l|l|l|l|l|l|l|}
\hline & 2002 & 2003 & 2004 & \multicolumn{2}{l|}{} \\
\cline { 2 - 7 } & $\mathbf{L R R}$ & $\boldsymbol{L R R}$ & $\boldsymbol{L R R}$ & $\boldsymbol{L R S P}$ & LRR & LRSP \\
\hline average \# article downloads/month [11] & 900 & 1800 & 3900 & 200 & 5200 & 790 \\
\hline total \# citations & 570 & 950 & 1500 & - & 2600 & $-[13]$ \\
\hline \# subscribers for email announcements & 625 & 670 & 705 & 80 & 750 & 160 \\
\hline
\end{tabular}

Table 1: Journal Usage

The second journal, Living Reviews in Solar Physics (LRSP, solarphysics.livingreviews.org, published by the Max Planck Institute for Solar Physics) was started in 2003 and covers all aspects of solar and heliospheric physics. Based on a similar publishing culture as within the relativity community and with LRR as a reference project, LRSP has made a successful start. Here we have noticed a great enthusiasm in making use of the multimedia-based features that an electronic journal can offer. In a field where scientists deal with a lot of visual material, the opportunity to incorporate hundreds of colourful images, movies, animations, and other types of resources provides a special asset. And only recently, the journal's editorial board suggested starting a (moderated) online commentary forum, where users can discuss and comment on published reviews. This feature is supposed to add another 'living' dimension to the journal.

The first two articles of LRSP were published mid 2004, ten more reviews were published in 2005. The article download numbers (see Table 1) confirm, despite the relatively low number of publications, already a substantial readership for the young journal.

With the second journal, we had to rethink many of the concepts, both technical and editorial, that had evolved over the years for LRR. For instance, the web site www.livingreviews.org, which was the initial home of LRR, was turned in the Living Reviews portal site, with individual journals accessible at $<$ subjectField $>$.livingreviews.org. The scheme for publication numbers needed to be revised, in order to set up a common URL resolver for all articles published in Living Reviews journals. Suddenly backward compatibility already became an issue, since we had to make sure that despite server restructuring, moving of files, HTML cleanup and new file naming conventions, the old URLs still remained valid. On the editorial side, slight changes with respect to the management of the refereeing process were adopted. We also had to think about questions of corporate identity for the journals appearance. In summary, the start of LRSP was a very important milestone and helped a lot in getting a better understanding of which aspects of the journal (in broadest sense technical, editorial, organizational) should be generalised, and which should be customisable to fit to community-specific needs. 


\subsection{Living Reviews in Social Sciences}

In summer 2004 two European-scale international research networks with some sixty participating institutions, funded by the European Union under its $6^{\text {th }}$ Research Framework were established. [14] Among other tasks, these two networks aim at taking stock of the research done in the field of European integration studies, focussing in particular on issues of efficient and democratic multi-level governance in Europe. This field is interdisciplinary, as contributing scholars come not only from the field of political science, but also from sociology and law. Alongside more traditional tools like a comparative research projects database and an online working paper series, the coordinators of the two networks finally supported the idea of trying out a new route of research stocktaking and dissemination that was never heard of before in the social sciences. It was a great opportunity that the Max Planck Society decided at this very moment to open up their Living Reviews concept and toolbox in order to offer it to "entrepreneurs" in other fields. Hence, the Living Reviews in European Governance (LREG, europeangovernance.livingreviews.org) is the first to be established in the social sciences.

It is important to note that the publishing culture in the social sciences is rather different from the natural sciences. First, the concept of reviews is not yet well established in political science in general, and in the field of European studies in particular. Reviews are not unknown, but are rare and we rather see them as an addendum to traditional journals that focus on original research papers. Second, online publishing is not common at all. The first E-Journal in the field (the European Integration online Papers - EIoP, [15]) started in 1997, but is, despite its success, still the only one. Although many institutions publish online working paper series, it is - in contrast to physics and other fields - not at all the rule to publish everything as an E-pre-print before sending it to a traditional journal, whether online or not. But the tides are slowly changing. Webpages such as Jstor [16] or Ingenta [17] show the formidable expansion of online sources for the Social Sciences. Web-portals or catalogues soon became attractive in order to provide researchers with easy access and some guidance. In the field of European Integration studies, the most common sources are the European Research Paper Archives (ERPA, [18]) and the Archive of European Integration (AEI, [19]). However, bibliographic databases and keyword/full text search can offer only minimal insights into deeper levels of the research or structured knowledge. The main problem thus has become one of dealing in a systematic and cumulative way with such a wealth of resources.

Hence, there are good reasons to believe that the idea of state-of-the-art reports in general, and of electronically published living reviews in particular may contribute to solving the problem as perceived by a growing number of scholars in the field. It was encouraging that the Living Reviews concept has been welcomed not only by the coordinators of the two networks, but virtually by all who have already heard of the initiative. In particular, already a dozen of first authors have accepted the invitation to contribute, thus showing their support of the idea. Transferring the Living Reviews concept to the social sciences is, however, no trivial task and needed, on the one hand, long discussions and initiatives to convince the right people (board members, authors, referees) and, on the other hand, a number of adaptations of the original concept and technology. In both respects, the editorial office, based in Vienna at the Political Science Department of the Institute of Advanced Studies, has played a key role in managing this process.

As regards marketing for the concept, it is important to note that the concept is not as straightforward as it may seem in the first place. It is perhaps not by chance that there are hardly any examples in the field to compare it to: the research culture in the social sciences is not "cumulative" in the sense that each and every research result fits into a growing and structured, well-interconnected and tested "body of knowledge". In this respect, establishing the concept of state-of-the-art reviews is almost tantamount to a "revolution" in this research field. Therefore, the concept requires much explanation and persuasion of potential board members, reviewers and especially authors. One important task is to convince authors to read all editorial policies and guidelines with a view to understanding the complexity of the concept. In addition, it was necessary for the respective editorial board member to explain the concept verbally as part of the first contact. Our experience shows that such a procedure is successful. However, potential misunderstandings and misinterpretations occur nonetheless. Pointing these cases, the Editorial Team is asked to provide help and further explanations. A task that still lies ahead of us is to ensure that the new and innovative concept will be accepted by our potential readers, the research community.

As regards the necessary adaptations, we may distinguish first, textual and procedural, and second, technical adaptations. Concerning the various descriptive texts and procedural rules, including the authoring guidelines and refereeing procedures, they needed new wording to conform to what is common in the social sciences. Also, the procedures had to be fixed in a precise manner in anticipation of potential conflicts when it comes to checking the adequacy of state-of-the-art reports in a field that is not used to producing cumulative knowledge and that is based on very different approaches of looking at its objects of study. 
Furthermore, the necessary technical infrastructure had to be set up in Vienna. This also includes the LREG logo and website (see europeangovernance.livingreviews.org). In general, LREG plans to apply all features and tools as developed for the original Living Reviews. However, the Living Reviews software has been developed in the framework of physics. This means that some features are not easily transferable to the social sciences. To take but the most obvious example, political scientists write their texts almost universally with the text editor WINWORD from Microsoft, not with LaTeX; they are not used to the bibliographic format BibTeX, but use tools like EndNote or similar products instead. As the original software is tuned to prepare the online articles on the basis of LaTeX files, we needed to find a way to allow our authors to submit the reviews in their usual format and to transform it in the editorial office to feed it into the LR software. Our interim solution is that the Managing Editor has built up LaTeX competencies and will do the necessary conversion in a semi-automated way. As regards the download format from the bibliographic database, the respective tool has to be adapted in order to produce EndNote files instead. As the software is quite flexible and the problems are minor, we are optimistic that the software will be able to produce Living Reviews that conform to the needs of the social sciences. As LREG is the first such journal outside physics, future journals will benefit from our experience and the technical adaptations already made.

Since September 2004, LREG has its homepage, all general documents have been prepared, an editorial board with three topic editors and a well-functioning editorial office have been established, a list of subjects and titles as well as potential authors of reviews has been agreed upon, and more than fifteen authors have already accepted to contribute and are working on their reviews. Now, in spring 2006, LREG is on the brink of publishing the first Living Reviews in the field. The very first manuscript has already been fed into the refereeing process, and other manuscripts will follow shortly. Although it is certainly too early to draw any definitive conclusions, we feel confident enough to come up with the following suggestions and recommendations for those interested in setting up a similar journal in another field of the social sciences:

- Such a project should be based on broad consensus (as was the case for LREG where two major research networks endorsed the concept). Nevertheless, even given such consensus at the beginning, there is much need for constant efforts to explain the concept, to convince and to motivate during the initial years.

- As regards the definition of the scope of the journal and the individual subjects, we come to the conclusion that starting too broad implies the risk of becoming elusive and starting too narrow may lead to difficulties in attracting enough attention and publicity; hence the subject fields must be well defined and comprehensive (LREG started with the core research fields and is open to expanding its coverage at a later stage).

- Funding should be guaranteed for several years as setting up a Living Reviews journal is not a quick task, but takes a lot of time, especially in a new discipline; our experience shows that it takes at least one and a half years from start to the first published review (in the case of LREG, funding is guaranteed for four years - which may be too short; further funding might be dependent on the success of the concept).

In conclusion, the editorial board feels confident that the quality of the first LREG articles, together with the reputation of the authors, will contribute to a modern publication culture that has authoritative reference points as regards the achievements, results and shortcomings of previous research. We hope that we will reach our prime goal, which is that, after some five to ten years, LREG articles will have become the standard reference in European Governance research. Although the concept has been unknown so far, we expect that the main features of the Living Reviews concept will also be convincing in the social sciences at large (e.g. the fact that this resource will always be up-to-date; that it cites all important references; and that it points at research gaps). The fact that the first Living Reviews journal (LRR) has been recently accepted in the Science Citation Index (SCI) is encouraging for LREG, too. Although much disputed, the Social Science Citation Index (SSCI) is still an important point of reference in the social sciences as well. A realistic goal for the mid-term future is that the concept will flood into neighbouring research fields (like Political Theory, International Politics etc.). In the long run, once the social sciences communities can actually see the attractiveness and usefulness of the concept, Living Reviews may well become a major success in the whole area.

\subsection{Living Reviews in Landscape Research}

In an advanced stage of preparation is Living Reviews in Landscape Research (LRLR, landscaperesearch.livingreviews.org). It will be published by the Leibniz-Centre for Agricultural Landscape Research (ZALF) and focuses on evaluating the state of the art as well as actual problems and future needs in interdisciplinary domains of landscape research. Particular emphasis is given to review articles identifying research needs and highlighting integration of disciplinary fragmented research on overlapping topics. LRLR will give a summarising voice to purposely integrated landscape research and thus supplement existing disciplinary journals covering this field. The subjects integrated by landscape research range from strict social 
sciences (population dynamics, migration research) across economics (production costs, prices and rents of land properties) to physical (climate change, soil erosion) and life science (habitats, interaction of species). Scenarios on possible developments of entire landscapes can only be achieved by scientists of the mentioned disciplines combining different scales and approaches. Review articles thus are a special challenge as well as a special benefit for any further merging of disciplinary work. Thus, the variety in publication culture among the three living review journals mentioned above has to be combined harmonically in LRLR. From this, besides the synergy effects on the research subject of landscape, synergy is also expected by merging different cultures of scientific publishing. The LRLR homepage has been released in September 2005. It offers basic information about the LRLR editorial office at ZALF, the board of editors-in-chief, the journal scope and provides an example of a full paper to also demonstrate the online performance on article level. Completing the board of topic editors and attracting articles is in progress.

\section{Lessons Learned - Hints for Future "Cyber activists"}

Innovation in publishing heavily depends on accessibility, the degree of awareness, the quality control and the speed and cost of publications. A well functioning and structured Open Access system like the Living Reviews has the chance to offer these conditions, as the following key factors will show. Besides the description of these key factors and how we met them, we will also compile a few more practical hints that may help people when considering starting up their own Living Reviews journal.

\subsection{Quality Control}

Inevitably the quality of the published content is the most important criterion for whether a journal will become a success or not. The quality of published content in Living Reviews is guaranteed by various factors: international editorial boards commission articles from well-known experts in the fields, and classical peerreview mechanisms are applied before an article is published. The peer-review process is tailored to the needs of each journal and applies methods according to the common standard in the respective field. We keep an eye on current developments to open 'classical' peer review to more transparent approaches to evaluate the quality of publications, but so far no attempts for alternative quality control mechanisms for Living Reviews articles have been made. It is felt that open peer review may not be so well applicable to solicited articles, and that authors may have reservations in this case.

\subsection{Open Access}

When the first journal, Living Reviews in Relativity, was started, 'open access' was not yet an established term. However, it was clear from the very beginning, that only low access barriers can help establishing a young startup-journal with a new concept to increase readership. The reasons for choosing a free web-based dissemination format are today the same as at that time: a potential readership only limited by the number of available internet connections, its availability for every search and retrieval tool,, making current research immediately accessible (also to scientists whose institution may not be able to afford expensive journal subscriptions), increasing research impact. With the first journal, LRR, being online in its ninth year by now, Living Reviews can rightfully be considered as a senior project in the Open Access movement. Living Reviews was listed as an example for a 'new generation of OA journals' when the Budapest Open Access Initiative was launched, and the editor-in-chief of LRR was among the initial signatories of the initiative [20].

There are hints that the open access approach is at present reaching a new level of acceptance and support. The largest German research funding agency (DFG, German Research Foundation) agreed in January 2006 to combine future financial support with the suggestion to DFG-funded researchers to publish via open access (instead or in addition to publication in subscription journals, [21]). In addition, the Austrian Science Fund (FWF) for example has committed them to funding all refereed articles in Open Access Journals that are outcomes of funded projects [22].

Another indicator for increasing dissemination of scientific information via open access is the press release from the Directory of Open Access Journals [23] dated January 13, 2006, reporting that this portal now contains 2000 quality controlled scientific and scholarly electronic journals that are freely available on the web. The Directory of Open Access Journals, run by the library of the University of Lund, Sweden, and emerged directly from the Budapest Initiative on Open Access, listed less than 700 OA-journals just one and a half year ago.

Finally, OA-journals have entered into the Journal Citation Report and related bibliometric products of ISI Thomson. The Journal Citation Report of 2003 (released June 2004) listed 239 OA-journals. For 197 of them, an Impact Factor already could be computed and published [24]. The year before (2002), just 192 OA-Journals 
were listed in the Journal Citation Report [25]. However, the majority of the annual increase from 192 to 239 ISI-listed journals was due to established subscription journals that shifted their politics to OA, and not due to recently founded OA-journals. The ranking of journals by citations today is supplemented by rankings via counts of downloads or hits and by findings by search engines. Thus, new ranking indicators relating to online means of article reference might soon gain general acceptance [26]. To sum up, presently a lot of activities catalyse the open access movement and we believe that with Living Reviews we have just chosen the right road.

\subsection{Visibility and Accessibility}

The integration in established (community-specific and global) information systems is mandatory for a journal project. We regard it as a big success that Living Reviews has meanwhile achieved high Google ranking for its journal contents. Immediately after individual publications, journal contents are indexed by Google. Even a user searching for very generic terms like 'solar physics', 'European governance' or 'relativity' gets on the first page of search results the links to our journals. Of course, the readers of our articles may not be searching for such general keywords, but it is also clear that Google increasingly complements or even replaces traditional tools for a scientist's information retrieval (see [26]). This can be confirmed by the search query report in our web statistics [12], where very detailed requests (like 'wheeler deWitt equation') to Google led users to our site.

The journals are abstracted in ADS, OAIster, Zentralblatt MATH, the Directory of Open Access Journals. RSS feeds are available for journal contents. Wand we encourage authors to post articles before the publication in institutional repositories. On a more informal basis, it very interesting to see a web of individual recommendations (like links from scientists homepages) and references evolving around the journals. We have already found blog discussions regarding journal contents or concept, and third parties have listed LRR as a 'cool site' in the DMOZ project [27]. In conclusion - the journals are very well integrated in the worldwide knowledge space.

\subsection{Get the Right People}

Crucial for the endeavour of Living Reviews is to ask and get the right people - editorial board and ream members, authors and referees.

Endorsing Living Reviews means first of all persuading senior scientists to become members of the editorial board and thus helping to increase the reputation of the journal. In a next step, their basic reputation is the crucial factor of an active recruitment of reliable and high-ranking authors. The board has to develop the scope of the journal very carefully, and to introduce topics that are not only important but also progressing in the foreseeable future, so that a 'living' review is the appropriate format to report about it. All subjects should show active research. The "Living" aspect of the journal assumes that subjects evolve enough in a couple of years to warrant updating the review.

In general, authors should be able to survey recent work, summarize important results, evaluate the significance and inter-connections of these results, assess where new development is needed, and provide entry points into the essential literature and into web sites and other useful electronic resources. Regarding this, our experience has shown that the liability of Living Review authors is enhanced when their current academic status is neither at the top nor at the bottom level of academic careers. The burden of writing an innovative article like a Living Review might be too much for a young $\mathrm{PhD}$ candidate. In contrast, the high-ranking professors often have too many responsibilities and commitments already, to be able to take upon them writing and maintenance of such a review. Authors should have, as far as can be predicted, still quite a number of years in active research ahead of them to make sure that the review will get updated a couple of times.

When planning a journal release, one should consider the timescales carefully. According to our experiences, the journal start-up phase, namely the recruitment of an editorial board and the shaping of a well-thought-out concept for the journals coverage, takes about two years. Furthermore, one should take into account the special dynamics of authoring review articles. Usually the review article is just an additional task to authors' over-full schedule of academic commitments and original research, therefore writing an article takes in most cases at least one year. The first review(s) published should undergo an internal board refereeing procedure to ensure that the manuscript corresponds with the Living Review features, contents and styles. Once a review is published, it could serve as a template for all other authors.

It has proven useful if the editorial team is organised within one institution (University, Research Department, etc.). The editorial board and team should act as a forum where authors can pose any questions they might have. It is important to guide authors through the process of writing very carefully (especially when the concept is new in the academic discipline). 


\subsection{Being Always Up-To-Date}

In the last decades the scholarly sources available in print and electronic format has grown tremendously. The information overload is common to all fields of current science. The selection of sources is therefore a delicate issue. Either we get lost in an ever-strengthening avalanche of novel data and ideas or enormous chunks of knowledge remain unconsidered. With the concept of Living Reviews, we have tried to bring more structure and guidance into this world of 'information overload'. As a research tool for the entire scientific community it is not just an entry point for further reading and research, but also a point of reference that can be consulted time and again to stay up to date with the current research. It provides an effective qualitative filter and systematic evaluation on the latest developments. Hence the relevant data or materials should not be any more invisible or inaccessible to those who desperately need it. Borrowing Thomas Kuhn's concept of paradigm [28], Living Reviews try to publish state-of-the-art articles in a less chaotic but more 'paradigmatic' manner. An authoritative selection of sources, like the Living Reviews, thus becomes an indispensable tool in order not to be overcome by the ever-expanding supply of sources.

\section{6 ... Get Started!}

It is the goal of the Living Reviews project to facilitate the foundation of Living Reviews journals in other areas of research. Potential journal founders can not only benefit from our technical developments, but also from the extensive knowledge base built up in many years of developing, starting, and running electronic journals. We invite other research communities to explore the journal concept and its applicability to their field and will be happy to welcome further siblings in the Living Reviews journal family.

\section{Acknowledgements}

We wish to thank the Heinz Nixdorf Foundation for supporting the core Living Reviews project at the Heinz Nixdorf Center for Information Management in the Max Planck Society; the European Union for supporting the two research networks CONNEX and NEWGOV, which has made it possible to found the first Living Reviews journal in the social sciences; Vera Osswald and Frank Schulz (from the LivRev team) and Lisa Hunt (IHS Vienna) for helpful discussions and advice.

\section{References and Notes}

[1] Living Reviews Portal [online]. Munich, Germany : Max Planck Society. URL http://www.livingreviews.org/, 2006-02-27.

[2] In terms of article length, their size varies between 25 and more than 400 print pages.

[3] Living Reviews Software Development [online]. Munich, Germany : Max Planck Society. URL http://dev.livingreviews.org/, 2006-02-27.

[4] Heinz Nixdorf Center for Information Management in the Max Planck Society [online]. Munich, Germany : Max Planck Society. URL http://www.zim.mpg.de, 2006-02-27.

[5] FORKEL, R. Making a Home for a Family of Online Journals: The Living Reviews Infrastructure, in preparation, contribution to ELPUB2006.

[6] Main developers of the very idea of a 'living' review are Prof. Bernard SCHUTZ, director of the Max Planck Institute for Gravitational Physics and editor-in-chief of LRR, and Dr. Jennifer WHEARY, the first managing editor of LRR.

[7] arXiv.org e-Print Archive [online]. Ithaca, NY, U.S.A. : Cornell University. URL http://arxiv.org/, 2006-02-27.

[8] Two articles in the Journal of Electronic Publishing [9, 10] describe in detail the considerations and challenges while shaping the original format.

[9] WHEARY, J.; SCHUTZ, B. F. Living Reviews in Relativity: Making an Electronic Journal Live. $J$. Electron, 1997, publ. 3, no. 1. URL http://www.press.umich.edu/jep/03-01/LR.html, 2006-02-27

[10] WHEARY, J.; WILD, L.; WEYHER, C.; SCHUTZ, B. F. Living Reviews in Relativity: Thinking and Developing Electronically. J. Electron, 1998, publ. 4, no. 2. URL http://www.press.umich.edu/jep/0402/wheary2.html, 2006-02-27.

[11] Detailed statistics on web server traffic are available at [12].

[12] Living Reviews Journal Usage Statistics [online]. Munich, Germany : Max Planck Society. URL http://www.livingreviews.org/journalUsage.html, 2006-02-27. 
[13] No data available yet.

[14] CONNEX (Connecting Excellence on European Governance) and NEWGOV (New Modes of Governance).

[15] European Integration online Papers (EIoP) [online]. Austria : Austrian European Communities Studies Association. URL http://eiop.or.at/eiop/, 2006-02-27.

[16] JSTOR - The Scholarly Journal Archive [online]. URL http://www.jstor.org, 2006-02-27.

[17] INGENTA [online]. URL http://www.ingentaconnect.com, 2006-02-27.

[18] ERPA - European Research Papers Archive [online]. URL http://eiop.or.at/erpa/, 2006-02-27.

[19] Archive of European Integration [online]. Pittsburgh, U.S.A. : University of Pittsburgh. URL http://aei.pitt.edu/, 2006-02-27.

[20] The Budapest Open Access Initiative [online]. URL http://www.soros.org/openaccess/, 2006-02-27.

[21] DFG. DFG verabschiedet Open Access Richtlinien. Info Wissenschaft, 30 January 2006, no.4. URL

http://www.dfg.de/aktuelles_presse/information_fuer_die_wissenschaft/andere_verfahren/info_wissens chaft_04_06.html, 2006-02-27.

[22] FWF. Freiwillige Selbstverpflichtung zu Open-Access-Publikationen, URL http://www.fwf.ac.at/de/aktuelles_detail.asp?N_ID=125, 2006-02-27.

[23] DOAJ. The Directory of Open Access Journals reaches an important milestone: Now they are 2000 journals in the DOAJ [online]. URL http://www.doaj.org/articles/060113, 2006-02-27.

[24] The Thomson Corporation, Open Access Journals in the ISI Citation Databases: Analysis of Impact Factors and Citation Patterns. A citation study from Thomson Scientific, October 2004. Portable Document Format. URL

http://scientific.thomson.com/media/presentrep/essayspdf/openaccesscitations2.pdf, 2006-02-27.

[25] The Thompson Corporation. The Impact of Open Access Journals. A Citation Study from Thomson ISI, 2004. Portable Document Format. URL

http://scientific.thomson.com/media/presentrep/acropdf/impact-oa-journals.pdf, 2006-02-27.

[26] ROWLANDS, I.; NICHOLAS, D. New Journal Publishing Models: An international Survey of Senior Researchers. A CIBER report for the Publishers Association and the International Association of STM Publishers, 22 September 2005. Portable Document Format. URL http://www.slais.ucl.ac.uk/papers/dni-20050925.pdf, 2006-02-27.

[27] DMOZ. Open Directory - Science: Physics: Relativity: Journals and Preprints. Open Directory Project [online]. URL http://dmoz.org/Science/Physics/Relativity/Journals_and_Preprints/, 2006-02-27.

[28] KUHN, Thomas. The Structure of Scientific Revolutions. Chicago/London : The University of Chicago Press, 1964. 